\title{
Comentario de Jurisprudencia del Tribunal de Justicia de la Unión Europea
}

\author{
David Ordóñez Solís \\ Magistrado, Doctor en Derecho \\ y miembro de la Red de Expertos en Derecho de la Unión Europea
}

\begin{abstract}
Sumario: I. Introducción.-II. Primera parte. Los derechos de los ciudadanos: derechos tradicionales y nuevos derechos. 1. Los derechos de los ciudadanos en el Derecho de la Unión Europea. 1.1. El reconocimiento mutuo y el «turismo del permiso de conducir» en la Unión Europea. 1.2. Los derechos sociales: vacaciones retribuidas e igualdad. 1.3. La no discriminación de los nacionales de terceros Estados. 2. Los derechos fundamentales, los derechos de autor e Internet. 3. Los derechos del consumidor, en relación con el medioambiente y la contratación pública.-III. Segunda parte. La jurisprudencia del Tribunal de Justicia y el Derecho español. 1. Las sentencias prejudiciales «españolas». 2. Los nuevos reenvíos prejudiciales españoles: los asuntos del «céntimo sanitario» y sobre la protección de datos. 2.1. Los impuestos especiales sobre hidrocarburos y las Comunidades autónomas («céntimo sanitario»): asunto Transportes Jordi Besora. 2.2. La profundización en el derecho a la protección de los datos personales en Internet: asunto Google Spain.-IV. Relación de las sentencias comentadas.
\end{abstract}

\section{Introducción}

La actuación de un tribunal de justicia se legitima por la sensibilidad con que se pronuncie en la protección efectiva de los derechos de los ciudadanos. Precisamente y en la Unión Europea el Tribunal de Justicia va modelando su jurisprudencia con la inestimable ayuda y creatividad de los jueces nacionales que, pensando en los derechos de los ciudadanos, no dejan de explorar nuevas vías de protección.

A la vista del Informe del Tribunal de Justicia correspondiente a 2011, en el Tribunal de Justicia se registraron 688 asuntos, de los cuales 423 fueron reenvíos prejudiciales, es decir, a través del mecanismo de colaboración entre los jueces nacionales y el Tribunal de Justicia, que, como es notorio, ha permitido deducir los principios esenciales del Derecho de la Unión. Piénsese, por ejemplo que en 1961 se planteó la primera cuestión prejudicial del Derecho comunitario y es al poco tiempo cuando el Tribunal de Justicia inicia su fecunda jurisprudencia sobre el efecto directo y la prima- 
cía; algo que no se había producido en las 68 primeras sentencias del Tribunal de Justicia que, por simplificar, seguían la pauta tradicional de las organizaciones internacionales clásicas. A partir de 1961 el Tribunal de Justicia auxilia a los tribunales nacionales para resolver casos concretos, derechos reales y efectivos que afectan al ciudadano y no conflictos institucionales o interestatales, ciertamente importantes, pero que no tienen la trascendencia jurídica de la resolución de los casos que afectan a los ciudadanos.

En un segundo plano quedan en 2011 los recursos directos habiéndose registrado 81 de los que, en su mayoría, 73, son recursos por incumplimiento iniciados por la Comisión Europea contra los Estados miembros. Por tanto, como subraya el propio Tribunal de Justicia, siguen disminuyendo los conflictos interinstitucionales, fundamentalmente, recursos de anulación. Asimismo, se completa el total de asuntos de que conoció el Tribunal de Justicia en 2011 con los 162 recursos de casación, los 13 recursos de casación especiales, los 9 procedimientos especiales y los 3 procedimientos cautelares.

La otra preocupación estadística de los informes anuales del Tribunal de Justicia es la duración de los procedimientos. Así, una cuestión prejudicial tardó en resolverse unos 16 meses. No obstante y en supuestos especiales se ha conseguido que se tramiten rápidamente algunos asuntos (procedimiento prejudicial de urgencia, resolución prioritaria, procedimiento acelerado, procedimiento simplificado y posibilidad de juzgar sin conclusiones del Abogado General). El procedimiento prejudicial de urgencia se solicitó en 5 asuntos pero el Tribunal de Justicia solo accedió en dos supuestos lo que permitió terminar el procedimiento en dos meses y medio. También 30 asuntos prejudiciales se resolvieron mediante auto. En fin, ya casi la mitad de las sentencias se dictaron sin necesidad de que se pronunciaran conclusiones del Abogado General.

Me propongo, por tanto, analizar la jurisprudencia del Tribunal de Justicia en los cinco primeros meses de 2012 para lo cual analizo en una primera parte algunas sentencias muy vinculadas a los nuevos y a los tradicionales derechos de los ciudadanos consagrados por el Derecho de la Unión; y en una segunda parte me refiero a la misma cuestión desde la perspectiva del Derecho español repasando las sentencias dictadas a instancia de los jueces españoles así como algunos aspectos relevantes de los asuntos españoles registrados en el Tribunal de Justicia en lo que va de 2012.

\section{Primera parte. Los derechos de los ciudadanos: derechos tradicionales y nuevos derechos}

Resulta una obviedad señalar al Tribunal de Justicia como el responsable de nuevas perspectivas en la protección de los derechos de los ciu- 
dadanos. Y a tal efecto, el Tribunal de Justicia actúa interpretando ámbitos tradicionales del Derecho de la Unión, como es el caso de la política social, o nuevos como el relativo al espacio de libertad, seguridad y justicia. Asimismo y en los últimos años el Tribunal de Justicia ha desarrollado una decisiva jurisprudencia sobre la ponderación procedente cuando están en juego derechos fundamentales y derechos de autor. En fin, también el Tribunal de Justicia sigue reiterando la dimensión europea de ámbitos tan trascendentales como la protección del consumidor, el medio ambiente o la contratación pública.

\section{Los derechos de los ciudadanos en el Derecho de la Unión Europea}

Europa se ha hecho para los ciudadanos y, a pesar de las críticas, a veces fundadas, de desviaciones de tal meta, el Tribunal de Justicia no hace más que recordárnoslo mediante sentencias que resuelven problemas cotidianos. Me refiero a tres ámbitos bien distintos como son el permiso de conducir vehículos y su validez en otros países de la Unión Europea, los derechos sociales y los derechos de los nacionales de terceros países.

\subsection{El RECONOCIMIENTO MUTUO Y EL «TURISMO DEL PERMISO DE CONDUCIR»}

En la interpretación de las Directivas 91/439/CEE y 2006/126/CE sobre el permiso de conducción el Tribunal de Justicia ha establecido las excepciones admisibles en relación con el principio general de reconocimiento recíproco de estos permisos de conducir. Aun a riesgo de fomentar un turismo del permiso de conducir, el Tribunal de Justicia subraya la importancia del reconocimiento recíproco de los permisos expedidos por los distintos Estados miembros de la Unión.

En la sentencia Akyüz (C-467/10) se plantea si un ciudadano alemán al que en Alemania se le denegó la expedición del permiso de conducir por no cumplir los requisitos físicos y mentales para la conducción segura de un vehículo, podía hacer uso del permiso obtenido en la República Checa.

Frente a los argumentos de las autoridades alemanas, el Tribunal de Justicia mantuvo, por una parte, que un Estado miembro no puede establecer los límites mínimos o máximos de aptitud para reconocer el permiso de conducir concedido en otro Estado miembro. A tal efecto, el Tribunal de Justicia puntualiza: «permitir que el Estado miembro de acogida no reconozca un permiso de conducción expedido en otro Estado miembro, basándose en que al titular de dicho permiso se le denegó la expedición de un primer permiso de conducción en el primer Estado y en que el Estado miembro de expedición no comprobó si los motivos que dieron lugar a di- 
cha denegación de expedición dejaron de existir, tendría como efecto que el Estado miembro que hubiera establecido los requisitos más estrictos para la expedición de un permiso de conducción podría determinar el nivel de exigencias que deberían respetar los otros Estados miembros para que los permisos de conducción expedidos en estos últimos pudieran ser reconocidos en su territorio» (apartado 56).

Aunque, por otra parte y con el fin de atajar cualquier tipo de fraude, el Tribunal de Justicia considera admisible que «un Estado miembro de acogida deniegue el reconocimiento en su territorio del permiso de conducción expedido en otro Estado miembro, cuando se demuestra, no en función de la información de que dispone el Estado miembro de acogida, sino sobre la base de las indicaciones que figuran en el propio permiso de conducción o en otras informaciones incontestables procedentes del Estado miembro de expedición, que el requisito de residencia normal [...] no se cumplió» (apartado 62).

En la sentencia Hofmann (C-419/10) se plantea la misma cuestión de un ciudadano al que se le retira en Alemania el permiso de conducir y lo obtiene en la República Checa.

El Tribunal de Justicia subraya que la Directiva 2006/126 introduce un cambio respecto de la anterior Directiva 91/439 y, por tanto, constata la voluntad del legislador de la Unión de reforzar el principio de unicidad de los permisos de conducción y evitar que una persona cuyo permiso de conducción esté restringido, suspendido o retirado en un Estado miembro pueda conseguir que se le expida un permiso de conducción en otro Estado miembro o que se le reconozca la validez de tal permiso, es decir, se trata de combatir el «turismo del permiso de conducir» (apartados 70 a 73).

Sin embargo, esto no le impide al Tribunal de Justicia insistir en la necesidad de aplicar el principio de reconocimiento mutuo de modo que «al haber expedido las autoridades checas el permiso al Sr. Hofmann el 19 de enero de 2009 [...] una vez finalizado el período de prohibición para solicitar un nuevo permiso que acompañaba a la medida de retirada de permiso dictada en Alemania contra el interesado, las autoridades alemanas no están facultadas para denegar el reconocimiento de la validez del permiso expedido de este modo» (apartado 89).

\subsection{LOS DERECHOS SOCIALES: VACACIONES RETRIBUIDAS E IGUALDAD}

En el ámbito de los derechos sociales la interpretación de los principios fundamentales de no discriminación y de las directivas de armonización ha permitido al Tribunal de Justicia pronunciarse en relación con el derecho a las vacaciones, a la no discriminación por jubilación de trabajadores a tiempo parcial y por razón de sexo o de origen étnico. 
La sentencia Domínguez (C-282/10) se pronuncia nuevamente sobre la aplicación del derecho a las vacaciones en caso de baja por enfermedad. En este supuesto, Maribel Domínguez, contratada de un organismo público francés, sufrió un accidente in itinere y estuvo de baja laboral desde el 3 de noviembre de 2005 hasta el 7 de enero de 2007, pero reclamó las vacaciones que no pudo disfrutar durante ese periodo.

En primer lugar, el Tribunal de Justicia consideró que la regulación francesa que supeditaba el derecho a vacaciones anuales retribuidas a la existencia de un tiempo de trabajo efectivo mínimo de diez días o de un mes durante el período de devengo de tales vacaciones era contraria al Derecho de la Unión.

En segundo lugar, el Tribunal de Justicia puntualiza: «no puede restringirse el derecho a vacaciones anuales retribuidas de al menos cuatro semanas que tiene todo trabajador, con independencia de que éste haya estado de baja médica durante dicho período de devengo ya sea a causa de un accidente en el lugar de trabajo o en cualquier otro lugar, ya a causa de una enfermedad de la naturaleza u origen que sea» (apartado 30). Sobre este particular, el Tribunal de Justicia reitera la conocida jurisprudencia sobre el efecto directo, la interpretación conforme y la responsabilidad por infracción de las directivas.

Por último, el Tribunal de Justicia admite que los Estados miembros establezcan que el derecho a vacaciones anuales retribuidas varíe en función del origen de la baja médica del trabajador, siempre que sea igual o superior al período mínimo de cuatro semanas previsto por la Directiva 2003/88/CE relativa a determinados aspectos de la ordenación del tiempo de trabajo.

En la sentencia O'Brien (C-393/10) se plantea si la Directiva 97/81/ CE sobre el trabajo a tiempo parcial se aplica a una profesión tan particular como la de los jueces a tiempo parcial, en este caso en lo que se refiere a su jubilación. En el Reino Unido este tipo de jueces a tiempo parcial no tienen derecho a la jubilación y por eso el Tribunal Supremo británico le pregunta al Tribunal de Justicia si los jueces son trabajadores y si puede distinguirse entre jueces a tiempo completo y a tiempo parcial.

Sobre la primera cuestión, el Tribunal de Justicia aclara, por una parte, que «el hecho de que los jueces estén sometidos a modalidades de servicio y de que puedan ser considerados trabajadores [...] en modo alguno lesiona el principio de independencia del poder judicial ni la facultad de los Estados miembros de establecer un régimen jurídico específico que regule la magistratura (apartado 47). Y seguidamente da una serie de pautas que permitirían determinar si son trabajadores a tiempo parcial a los efectos de aplicar la Directiva (modalidades de nombramiento y de separación de los jueces, modo de organizar su trabajo, derecho a la indemnización por enfermedad, a las prestaciones por maternidad y por paternidad y a otras prestaciones si- 
milares). De modo que el Tribunal de Justicia considera que corresponde a cada Estado miembro determinar si los jueces están incluidos en el concepto de trabajador a tiempo parcial, «siempre que ello no lleve a excluir arbitrariamente a esta categoría de personas del beneficio de la protección brindada por la Directiva 97/81» (apartado 42).

En fin, la sentencia Meister (C-415/10) se refiere a la interpretación de la Directiva 2000/43/CE relativa al principio de igualdad de trato de las personas independientemente de su origen racial o étnico. En este caso la Sra. Galina Meister, nacida en 1961 y de origen ruso, denunció haber sido discriminada en Alemania por razón de su sexo, de su edad y de su origen étnico por una empresa que ni siquiera la había convocado a la entrevista de trabajo y, sin embargo, volvía a solicitar nuevos candidatos.

En este caso el Tribunal de Justicia se refiere a la regla de la carga de la prueba y considera que, por ejemplo, la discriminación indirecta puede acreditarse por cualquier medio, incluso basándose en pruebas estadísticas (apartado 43). Sin embargo, el ordenamiento de la Unión no prevé «el derecho de un trabajador que alega de forma verosímil que reúne las condiciones enunciadas en un anuncio de contratación y cuya candidatura no ha sido seleccionada de acceder a la información que indica si al término del proceso de selección el empresario ha contratado a otro candidato» (apartado 46).

\subsection{LA NO DISCRIMINACIÓN DE LOS NACIONALES DE TERCEROS ESTADOS}

El espacio europeo de libertad, seguridad y justicia constituye un ámbito especialmente propicio para la ampliación de los derechos de los nacionales de terceros países en la Unión.

La sentencia Kamberaj (C-571/10) es un ejemplo particularmente interesante donde se plantea la cuestión de la discriminación de ciudadanos de terceros países residentes en la Unión Europea. Se trata de un albanés que reside en Italia y tiene un empleo estable en Bolzano, siendo titular de un permiso de residencia de duración indeterminada. En esta provincia autónoma se establecen dos tipos de fondos: el destinado a los ciudadanos de la Unión y el previsto para los nacionales de terceros países. Al haber solicitado un ciudadano albanés residente de larga duración ayudas a la vivienda, se le deniegan por haberse agotado el fondo destinado a ciudadanos de terceros países.

A raíz de este litigio, el tribunal de Bolzano le plantea al Tribunal de Justicia numerosas cuestiones que el Tribunal europeo delimita y contesta de modo que, por una parte, comprueba que aun pueden persistir discriminaciones por razón de la nacionalidad en la Unión Europea; pero, por otra parte, el Tribunal de Justicia considera que en el caso de los nacionales de terceros países con permiso de residencia de larga duración no puede ser 
discriminados salvo en supuestos excepcionales previstos en la Directiva 2003/109 sobre la aplicación del principio de igualdad de trato de las personas independientemente de su origen racial o étnico.

Por tanto, el Tribunal de Justicia aplica la Directiva 2003/109 que equipara a los nacionales de terceros Estados residentes de larga duración con los ciudadanos de la Unión en cuanto se refiere a las prestaciones de la seguridad social, de la asistencia social y de la protección social tal como se definen en la legislación nacional y en el acceso a bienes y a servicios y el suministro de bienes y servicios a disposición del público, así como los procedimientos para acceder a la vivienda. No obstante, la Directiva permite a los Estados miembros limitar la igualdad de trato a las prestaciones básicas respecto de la asistencia social y la protección social. Y a la hora de aplicar la referida Directiva, el Tribunal de Justicia insiste en que es necesario aplicar la Carta de Derechos Fundamentales de la Unión, citando expresamente su artículo 34.3, de modo que, «con el fin de combatir la exclusión social y la pobreza, la Unión (y, por tanto, los Estados miembros cuando apliquen el Derecho de la propia Unión) "reconoce y respeta el derecho a una ayuda social y a una ayuda de vivienda para garantizar una existencia digna a todos aquellos que no dispongan de recursos suficientes, según las modalidades establecidas por el Derecho de la Unión y por las legislaciones y prácticas nacionales"» (apartado 80).

De este modo, los residentes de larga duración ya son más iguales, respecto de los ciudadanos de la Unión, en ámbitos bien precisos. Y, en consecuencia, el Tribunal de Justicia se dedica a defender la igualdad de los nacionales de terceros países con estatuto de residentes de larga duración en relación con los ciudadanos de la Unión. Y por eso el Tribunal de Justicia termina respondiendo al tribunal italiano: «la Directiva 2003/109 debe interpretarse en el sentido de que se opone a una normativa nacional o regional [...] que prevé, en relación con el reparto de los fondos destinados a la concesión de una ayuda a la vivienda, un trato diferente para el nacional de un país tercero que goza del estatuto de residente de larga duración, concedido de conformidad con las disposiciones de dicha Directiva, respecto del trato dispensado a los nacionales residentes en la misma provincia o región, siempre que la citada ayuda esté comprendida en una de las tres categorías contempladas en dicha disposición y que no resulte aplicable el apartado 4 de ese mismo artículo» (apartado 90).

\section{Los derechos fundamentales, los derechos de autor e Internet}

Sigue el Tribunal de Justicia pronunciándose sobre la ponderación de los derechos en juego en lo que se refiere a la protección de los derechos 
de autor e Internet y que, sin lugar a dudas, se completará con los reenvíos prejudiciales pendientes, incluido el planteado desde España y que analizo a continuación, y con el dictamen que la Comisión ha solicitado al Tribunal de Justicia sobre la compatibilidad del Acuerdo comercial contra la piratería y la falsificación (ACTA) con los derechos fundamentales de la Unión.

Entre tanto la sentencia Sabam / Netlog (C-360/10) constituye una reproducción de la sentencia, de 24 de noviembre de 2011, Scarlet Extended (C-70/10), y se refieren directamente a los límites que imponen los derechos fundamentales a la protección judicial de los derechos de autor. Se trata de profundizar en el problema jurídico planteado ya en la sentencia Promusicae y en el contexto del Tratado de Lisboa.

SABAM, la sociedad de autores de Bélgica, solicitaba al juez belga la adopción de una medida cautelar de cesación frente a Netlog, una empresa que explota una plataforma de red social en línea, con el fin de evitar el pirateo realizado por determinados internautas. El Derecho nacional invocado es una Ley belga que transpone las Directivas 2001/29 y 2004/48 y que, en particular, permite al juez nacional constatar la existencia y ordenar la cesación de cualquier atentado a un derecho de autor o a otro derecho similar para lo que puede adoptar una orden de cesación frente a los intermediarios cuyos servicios sean utilizados por un tercero para atentar contra tales derechos.

Sin embargo, el Tribunal de Justicia considera que en este supuesto es desproporcionada la obligación que se impone de filtrado y bloqueo de datos electrónicos. A tal efecto, el Tribunal de Justicia explica: «el requerimiento judicial implicaría una vulneración sustancial de la libertad de empresa del prestador de servicios, dado que le obligaría a establecer un sistema informático complejo, gravoso, permanente y exclusivamente a sus expensas, que además sería contrario a los requisitos recogidos en el artículo 3, apartado 1, de la Directiva 2004/48, el cual exige que las medidas adoptadas para garantizar el respeto de los derechos de propiedad intelectual no sean inútilmente complejas o gravosas» (apartado 46).

Del mismo modo, la sentencia Bonnier Audio (C-461/10) se refiere a la aplicación en el ámbito jurisdiccional civil de la Directiva 2006/24/CE sobre la conservación de datos adoptada para el ámbito penal pero en la misma el Tribunal de Justicia ilustra al Tribunal Supremo sueco sobre los poderes y los límites del juez nacional.

La empresa Bonnier Audio y otros editores de 27 audiolibros obtuvieron del Juzgado de Primera Instancia de Solna en Suecia un requerimiento judicial frente a usuarios de Internet que el 1 de abril de 2009 habían descargado archivos entre las 3:38 y las 5:45 horas que vulneraban sus derechos de propiedad intelectual. Sin embargo, el Tribunal de apelación de Estocolmo anuló el requerimiento de la entrega de datos, llegando el asunto en 
casación al Tribunal Supremo sueco que le preguntó al Tribunal de Justicia si se aplicaba la Directiva sobre conservación de datos.

En sus Conclusiones el abogado general Niilo Jääskinen niega terminantemente que la Directiva 2006/24/CE sobre la conservación de datos adoptada para el ámbito penal pueda aplicarse en materia civil. Y, además, añade: «los derechos fundamentales en materia de protección de datos personales y de la vida privada, por una parte, así como en materia de protección de la propiedad intelectual, por otra parte, deben disfrutar de una protección equivalente. Por tanto, no procede privilegiar a los titulares de derechos de propiedad intelectual permitiéndoles el uso de datos personales legalmente recogidos o conservados para fines ajenos a la protección de sus derechos. La recopilación y utilización de dichos datos para tales fines respetando el Derecho comunitario en materia de protección de datos personales requeriría la previa adopción por el legislador nacional de disposiciones detalladas, conforme al artículo 15 de la Directiva 2002/58» (apartado 62).

En su sentencia Bonnier Audio el Tribunal de Justicia corrobora esta opinión y señala que la Directiva 2006/24 constituye una normativa especial y claramente delimitada, que deroga y sustituye a la Directiva 2002/58, de alcance general (apartado 43). Sin embargo y en el mismo sentido que la sentencia Promusicae y el auto LSG-Gesellschaft zur Wahrnehmung von Leistungsschutzrechten, el Tribunal de Justicia considera que la legislación de la Unión Europea no se opone a que los Estados miembros establezcan una obligación de transmitir a particulares datos personales para permitir ejercer acciones ante la jurisdicción civil contra las infracciones al Derecho de propiedad intelectual, pero tampoco obliga a las legislaciones nacionales, como la sueca, a imponer tal obligación sino que permite adoptar un requerimiento judicial de comunicar los datos personales de Internet siempre que existan indicios reales de vulneración de un derecho de propiedad intelectual sobre una obra, que los datos solicitados puedan facilitar la investigación de la vulneración del derecho de autor y que el fin perseguido por dicho requerimiento sea más importante que el daño o perjuicio que se puedan causar a la persona afectada o a otros intereses contrapuestos.

Estos elementos de interpretación deben tenerse en cuenta en España a la hora de aplicar la Ley Sinde, en virtud de la cual los Jueces (Centrales de lo Contencioso-administrativo) y Tribunales Contencioso-administrativos (Salas de la Audiencia Nacional y del Tribunal Supremo) tienen el poder de requerir a los proveedores de acceso a servicios de Internet que notifiquen datos personales de los presuntos infractores o exigir a los mismos proveedores que retiren determinados contenidos de Internet.

La sentencia Marco del Corso (C-135/10) se refiere al alcance de los derechos de autor y, en particular, a la interpretación de la Directiva 92/100/ CEE sobre derechos de alquiler y préstamo y otros derechos afines a los de- 
rechos de autor en el ámbito de la propiedad intelectual. En el litigio principal se enjuiciaba la recaudación realizada por la sociedad italiana de gestión de derechos por las emisiones radiofónicas recibidas en la sala de espera de un dentista de Turín que se negaba a pagar canon alguno.

En primer lugar, el Tribunal de Justicia recuerda su jurisprudencia conforme a la cual las disposiciones del Acuerdo sobre los Aspectos de los Derechos de Propiedad Intelectual relacionados con el Comercio (ADPIC) carecen de efecto directo y tampoco confieren a los particulares derechos que estos puedan invocar directamente ante los tribunales en virtud del Derecho de la Unión (apartado 46).

Y, en segundo lugar, el Tribunal de Justicia resuelve el problema de la interpretación del concepto de «comunicación al público», a los efectos de la aplicación de la Directiva 92/100 «en el sentido de que no incluye la difusión gratuita de fonogramas en una consulta odontológica, como la del asunto principal, en el marco del ejercicio de una profesión liberal, a favor de los pacientes, que disfrutan de ella independientemente de su voluntad»; lo que supone que tal difusión no confiere a los productores de fonogramas el derecho a percibir una remuneración (apartado 102).

El examen del Tribunal de Justicia resulta muy interesante en la medida que compara el supuesto de la música en la consulta del dentista y la de los hoteles y los bares o restaurantes. Por lo que se refiere a los hoteles, el Tribunal señala que «la intervención del establecimiento hotelero para dar acceso a sus clientes a una obra radiodifundida es una prestación de servicios suplementaria efectuada con el objetivo de obtener algún beneficio en la medida en que la inclusión de este servicio influye en la categoría del hotel y, por tanto, en el precio de las habitaciones». Y respecto de los establecimientos de restauración, a juicio del Tribunal de Justicia, «se hace para atraer clientes y puede repercutir sobre el número de personas que frecuentan dicho establecimiento y, en definitiva, sobre sus resultados económicos» (apartado 90). En cambio, en el caso de «los pacientes de un dentista acuden a una consulta de odontología con el único objeto de ser atendidos, no siendo inherente a la asistencia odontológica la difusión de fonogramas. Acceden a determinados fonogramas, en función del momento de su llegada al consultorio y de la duración de su espera así como de la naturaleza del tratamiento que se les dispensa, de manera fortuita y con independencia de sus deseos. Por ello no puede presumirse que el conjunto de pacientes de un dentista sea receptivo respecto la difusión de que se trate» (apartado 98) y tal difusión no reviste carácter lucrativo (apartado 99).

La sentencia DR, TV2 Danmark (C-510/10) se refiere a la interpretación de la Directiva 2001/29/CE relativa a los derechos de autor y derechos afines en la sociedad de la información. En este supuesto se preguntaba si se aplicaba la excepción al derecho exclusivo del autor de la obra a su re- 
producción «cuando se trate de grabaciones efímeras de obras, realizadas por organismos de radiodifusión por sus propios medios y para sus propias emisiones».

Pues bien, la interpretación del Tribunal de Justicia llega a la conclusión de que se trata de dos requisitos equivalentes y que por tanto tienen carácter alternativo (apartado 56); por eso, «en el supuesto de que no pueda considerarse que el tercero en cuestión actúa "en nombre" del organismo de radiodifusión, debe apreciarse, en una segunda fase, si cabe considerar que dicho tercero actúa, al menos, "bajo la responsabilidad" de aquél» (apartado 62). Y lo relevante para determinar esta situación es que «frente a terceros - en particular los autores a los que pueda causarse un daño con la grabación irregular de su obra-, el organismo de radiodifusión esté obligado a reparar todo efecto perjudicial de las acciones y omisiones del tercero - por ejemplo, una sociedad de producción televisiva externa y jurídicamente independiente - relacionadas con la grabación en cuestión como si el propio organismo de radiodifusión hubiera llevado a cabo tales acciones y omisiones» (apartado 64). Y sin que sean relevantes otras relaciones como las artísticas o las editoriales de la producción de radiodifusión (apartado 65).

En fin, la sentencia SAS Institute Inc (C-406/10) la High Court de Londres preguntaba por la interpretación de la Directiva 91/250 en relación con la demanda de una empresa sueca titular de un programa informático y que se reclamaba a otra empresa, WPL, por la elaboración de un software sustitutivo del programa SAS.

En primer lugar el Tribunal de Justicia define el ámbito de protección de la Directiva: «ni la funcionalidad de un programa de ordenador ni el lenguaje de programación o el formato de los archivos de datos utilizados en un programa de ordenador para explotar algunas de sus funciones constituyen una forma de expresión de ese programa y, por ello, carecen de la protección del derecho de autor sobre los programas de ordenador en el sentido de esta Directiva» (apartado 46).

Y seguidamente el Tribunal de Justicia puntualiza: «el titular de los derechos de autor sobre un programa de ordenador no puede invocar el contrato de licencia para impedir que quien haya obtenido esa licencia determine las ideas y los principios implícitos en todos los elementos de ese programa cuando realiza las operaciones autorizadas por dicha licencia así como los actos de carga y desarrollo necesarios para la utilización del programa de ordenador, siempre y cuando no infrinja los derechos exclusivos de ese titular sobre tal programa» (apartado 59).

No obstante y en tercer lugar, el Tribunal de Justicia precisa: «Sólo a través de la elección, la disposición y la combinación de tales palabras, cifras o conceptos matemáticos puede el autor expresar su espíritu creador de manera original y obtener un resultado, el manual de utilización del pro- 
grama de ordenador, que constituye una creación intelectual» (apartado 67); por lo que podría constituir una vulneración del derecho de autor la reproducción, en un programa de ordenador o en un manual de utilización de ese programa, de algunos elementos descritos en el manual de utilización de otro programa de ordenador protegido por los derechos de autor (apartado 70).

\section{Los derechos del consumidor, en relación con el medioambiente y la contratación pública}

Ha sido muy relevante la interpretación del Tribunal de Justicia en relación con la protección del consumidor frente a las cláusulas abusivas. En 2012 el Tribunal de Justicia ha corroborado el amplio alcance de la Directiva 93/13/CEE sobre las cláusulas abusivas en los contratos celebrados con consumidores.

Así, la sentencia NFH (Oficina húngara de Protección del Consumidor) (C-472/10) interpreta la Directiva 93/13/CEE y se refiere, en particular, a los contratos de duración determinada («contratos de fidelidad») en los que se exigen al consumidor, después de la celebración del contrato, gastos no acordados inicialmente por las partes y en el ámbito de la telefonía.

En primer lugar el Tribunal de Justicia se considera competente para interpretar el concepto de cláusula abusiva y para proporcionar al juez nacional los criterios que debe aplicar al examinar una cláusula contractual a la luz de las disposiciones de la Directiva, entendiéndose que incumbe a dicho juez pronunciarse, teniendo en cuenta dichos criterios, sobre la calificación concreta de una cláusula contractual particular en función de las circunstancias propias del caso. En este supuesto el Tribunal de Justicia considera que una cláusula contractual que prevea una modificación del coste total del servicio que deba prestarse al consumidor resultaría abusiva si no se indica el motivo o el modo de variación de dicho coste y si no confiere al consumidor el derecho a rescindir la relación contractual (apartado 24).

Y seguidamente el Tribunal de Justicia distingue entre las acciones individuales y las acciones colectivas en defensa de los derechos de los consumidores. El principio que inspira estos dos tipos de acciones es el mismo: «el sistema de protección establecido por la Directiva se basa en la idea de que el consumidor se halla en situación de inferioridad respecto al profesional, en lo referido tanto a la capacidad de negociación como al nivel de información, situación que le lleva a adherirse a las condiciones redactadas de antemano por el profesional, sin poder influir en el contenido de éstas» (apartado 33). 
Respecto de las acciones individuales, el Tribunal de Justicia subraya que su objetivo, tal como se deduce de la Directiva, es que las cláusulas abusivas no vinculen al consumidor, en las condiciones estipuladas por sus derechos nacionales, al ser una disposición imperativa que trata de reemplazar el equilibrio formal que el contrato establece entre los derechos y obligaciones de las partes por un equilibrio real que pueda restablecer la igualdad entre éstas (apartado 34).

$\mathrm{Y}$ en cuanto a las acciones colectivas, las de cesación ejercitadas por motivos de interés público, implican por su carácter preventivo y finalidad disuasoria, que «las cláusulas de las CG [condiciones generales de la contratación] de los contratos celebrados con consumidores que sean declaradas abusivas en el marco de una acción de cesación ejercitada contra el profesional de que se trate [...] no vinculen ni a los consumidores que sean parte en el procedimiento de cesación ni a aquéllos que hayan celebrado con ese profesional un contrato al cual le sean de aplicación las mismas CG» (apartado 38). Esto, en definitiva, supone que «la aplicación de la sanción de nulidad de una cláusula abusiva con respecto a todos los consumidores que hayan celebrado, con el profesional de que se trate, un contrato al cual le sean de aplicación las mismas CG garantiza que dicha cláusula no vinculará a esos consumidores, y al mismo tiempo no excluye otro tipo de sanciones adecuadas y eficaces que prevean las normativas nacionales» (apartado 40).

Por eso cuando una cláusula haya sido declarada abusiva, los jueces deben «aplicar de oficio, también en el futuro, todas las consecuencias previstas por el Derecho nacional para que los consumidores que hayan celebrado un contrato al cual le sean de aplicación las mismas CG no resulten vinculados por dicha cláusula» (apartado 43).

En materia de medio ambiente y, más en particular, en la sentencia Inter-Environnement Wallonie ASBL (Decreto valón) (C-41/11) el Tribunal de Justicia considera en determinados supuestos muy tasados que el juez nacional puede restringir los efectos derivados de la anulación de una norma medioambiental nacional que sea contraria al Derecho de la Unión.

En este caso el Consejo de Estado belga conocía de un recurso contra un Decreto valón de 2007 por el que se modificaba el Código del agua; sin embargo, la anulación de este Decreto regional suponía dejar un vacío en la regulación medioambiental hasta que se aprobase un nuevo Decreto, tal como ocurrió en 2011, conforme al Derecho de la Unión. El Tribunal de Justicia precisa que «el objetivo de protección del medio ambiente [...] constituye uno de los objetivos esenciales de la Unión y reviste un carácter tanto transversal como fundamental» (apartado 57). Y seguidamente admite que de modo excepcional el juez nacional mantenga determinados efectos del decreto anulado siempre y cuando dicho acto nacional constituya una 
medida de transposición adecuada de la Directiva; la adopción y la entrada en vigor del nuevo decreto no permita evitar los efectos perjudiciales en el medio ambiente que se derivan de la anulación del acto impugnado; la anulación del acto impugnado tenga como consecuencia crear un vacío legal por lo que respecta a la transposición de la Directiva que resulte más perjudicial para el medio ambiente en el sentido de que dicha anulación supondría una menor protección y vulneraría incluso el objetivo esencial de la citada Directiva; y, en fin, que el mantenimiento excepcional de los efectos de tal acto solo cubra el tiempo estrictamente necesario para que se adopten las medidas que subsanen la irregularidad declarada (apartados 59 a 62).

La sentencia Inter-Environnement Wallonie ASBL (Orden bruselense) (C-567/10) responde a un reenvío prejudicial del Tribunal Constitucional belga que quería saber si un plan de ordenación del suelo constituía un plan o programa de los previstos en la Directiva 2001/42/CE relativa a la evaluación de los efectos de determinados planes y programas en el medio ambiente. Respondida positivamente la cuestión, el Tribunal de Justicia determina si la derogación total o parcial de este plan debía someterse a una evaluación medioambiental.

A tal efecto, el Tribunal de Justicia comprueba que la derogación, total o parcial, de un plan o de un programa pueda tener efectos significativos en el medio ambiente, puesto que la misma puede suponer una modificación de la planificación prevista en los territorios afectados (apartado 38) por lo que está incluido en principio en el ámbito de aplicación de la Directiva y está sometido a sus normas de evaluación medioambiental.

Por último, quiero referirme a dos sentencias sobre contratación pública, sobre la Directiva 2004/18/CE sobre coordinación de los procedimientos de adjudicación de los contratos públicos de obras, de suministro y de servicios.

En la sentencia SAG ELV Slovensko (C-599/10) se plantea el efecto de la petición de aclaración de ofertas en el marco de un litigio por la adjudicación de un contrato público, de más 600 millones de euros, relativo a la prestación de los servicios de cobro de peajes en las autopistas y en determinadas carreteras. A juicio del Tribunal de Justicia debe responder «en qué medida los poderes adjudicadores pueden o deben exigir aclaraciones a un candidato cuando estimen, en un procedimiento de licitación restringido, que la oferta de dicho candidato es anormalmente baja o imprecisa o que no se ajusta a las especificaciones técnicas del pliego de condiciones».

En su respuesta, el Tribunal de Justicia insiste en los principios de igualdad y de transparencia aplicados al procedimiento de adjudicación de contratos públicos. Y en cuanto a los supuestos de ofertas anormalmente bajas, el Tribunal de Justicia recuerda que el poder adjudicador tiene la obligación de formular claramente la petición dirigida a los candidatos afectados para 
que estos puedan justificar plena y oportunamente la seriedad de sus ofertas (apartado 31). Y en cuanto a las ofertas imprecisas o que no se ajusten a las especificaciones técnicas del pliego de condiciones, a juicio del Tribunal de Justicia el poder adjudicador «está obligado a tratar a los diferentes candidatos del mismo modo y con lealtad, de manera que, al término del procedimiento de selección de las ofertas y en vista de los resultados de éste, no pueda concluirse que la petición de aclaraciones benefició o perjudicó indebidamente al candidato o candidatos que la recibieron» (apartado 41). Esto, sin embargo, no impide que el órgano de contratación solicite aclaraciones siempre y cuando la petición de aclaración de la oferta se formule una vez que el poder adjudicador haya tomado conocimiento de la totalidad de las ofertas, la petición de aclaraciones se formule de manera equivalente para todas las empresas que se encuentren en la misma situación, si no existe un motivo objetivamente verificable que pueda justificar un trato diferenciado de los candidatos a este respecto, en particular cuando la oferta deba rechazarse en cualquier caso por otras razones; y, en fin, la petición de aclaraciones debe referirse a todos los puntos de la oferta que sean imprecisos o no se ajusten a las especificaciones técnicas del pliego de condiciones, sin que el poder adjudicador pueda rechazar una oferta por la falta de claridad de un aspecto de esta que no haya sido mencionado en esa petición (apartados 42 a 44).

En la sentencia Duomo Gpa y otros (C-357/10 a C-359/10) se suscita la cuestión relativa a las licitaciones convocadas por varios ayuntamientos italianos para la concesión de los servicios tributarios locales. En este caso se planteaba si estaba justificado que la legislación italiana exigiese de los licitadores, salvo las sociedades con participación estatal mayoritaria, haber desembolsado un mínimo de capital de 10 millones de euros para poder prestar un servicio de recaudación de tributos locales.

El Tribunal de Justicia precisa que en este caso todavía no se aplicaba la Directiva de servicios sino que había que interpretar y aplicar el Derecho primario, es decir, los principios de libre prestación de servicios y de la libertad de establecimiento del Tratado constitutivo. Después de constatar que la legislación italiana obstaculiza o hace menos interesante la libertad de establecimiento y la libre prestación de servicios, analiza si puede justificarse la legislación italiana atendiendo a una de las razones que recoge el Tratado constitutivo o puede considerarse, con arreglo a la jurisprudencia del Tribunal de Justicia, justificada por razones imperiosas de interés general.

Aun cuando se alegaba como motivo de justificación la protección de la administración pública contra el eventual incumplimiento de la sociedad concesionaria habida cuenta del elevado importe global de los contratos de que era titular, el Tribunal de Justicia consideró que la medida era despro- 
porcionada al existir ya determinadas precauciones previstas por la normativa italiana que protegían de un modo proporcionado a la administración frente a un eventual incumplimiento de los concesionarios.

\section{Segunda parte. La jurisprudencia del Tribunal de Justicia y el Derecho español}

Aunque España haya ganado importantísimas batallas jurídicas ante el Tribunal de Justicia y de especial trascendencia presupuestaria como ocurre en la sentencia España / Comisión (C-24/11 P) donde estaban en juego más de 113 millones de euros por correcciones financieras de ayudas del FEAGA al aceite de oliva en las campañas 1998 a 2001, en este comentario interesa más dar cuenta de las respuestas a cuestiones prejudiciales remitidas por los tribunales españoles y los más destacados reenvíos planteados y registrados ante el Tribunal de Justicia.

En 2011 y desde España se remitieron a Luxemburgo 27 reenvíos prejudiciales lo que confirma una tendencia al alza frente a una inicial reticencia de los jueces españoles y del propio Tribunal Constitucional que, felizmente, ha vencido su miedo y ha terminado planteando la primera cuestión prejudicial. En cambio, en 2011 la Comisión Europea solo interpuso 7 recursos por incumplimiento contra España.

\section{Las sentencias prejudiciales «españolas»}

Las sentencias pronunciadas por el Tribunal de Justicia en lo que va de 2012 reflejan el especial dinamismo de la jurisdicción civil en su vertiente mercantil y de la jurisdicción contencioso-administrativa.

La primera de las tres sentencias en materia civil, la sentencia Celaya Emparanza (C-488/10), se refiere al derecho exclusivo de utilización de un dibujo o modelo comunitario registrado. El Juzgado de lo Mercantil n. ${ }^{\circ} 1$ de Alicante y ${ }^{\circ}{ }^{\circ} 1$ de Marca Comunitaria había pedido al Tribunal de Justicia que interpretara los derechos exclusivos otorgados por dos modelos comunitarios registrados.

En el litigio se enfrentaban dos empresas que habían conseguido registrar dos dibujos o modelos comunitarios prácticamente iguales consistentes en un señalizador de viales para el tráfico rodado A tal efecto, el Tribunal de Justicia aplica el principio de prioridad «en virtud del cual el dibujo o modelo comunitario registrado anterior prima sobre los dibujos o modelos comunitarios registrados posteriores» (apartado 39). Y, asimismo, el Tribunal de Justicia le recuerda al juez mercantil español que el legislador de la 
Unión ha tomado efectivamente en consideración la buena fe para proteger al creador que no conocía el dibujo o modelo no registrado divulgado por su titular, pero el principio de prioridad no varía en función de la intención o del comportamiento de un tercero.

La sentencia González Alonso / Nationale Nederlanden Vida (C-166/11) supone la contestación al reenvío prejudicial de la Audiencia Provincial de Asturias sobre la Directiva 85/577/CEE de protección de los consumidores en el caso de contratos negociados fuera de los establecimientos comerciales, más en particular, respecto de la venta de seguros fuera de un establecimiento comercial.

La aseguradora Nationale Nederlanden había ofrecido en el lugar de trabajo del recurrente un producto financiero consistente en un seguro de vida a cambio del pago mensual de una prima destinada a ser invertida, en distintas proporciones, en renta fija, renta variable y productos de inversión financiera de la compañía aseguradora. La cuestión prejudicial pretendía determinar el ámbito de aplicación de la Directiva 85/577 y el Tribunal de Justicia llega a esta conclusión: «el legislador de la Unión, al adoptar la Directiva 85/577 y excluir de su ámbito de aplicación los contratos de seguro en su totalidad, consideraba contratos de seguro los contratos de seguro vinculados con fondos de inversión» (apartado 31). En consecuencia y en este supuesto no se aplica la Directiva 85/577.

En fin, la sentencia Banesto (C-618/10) constituye la respuesta del Tribunal de Justicia al reenvío de la Audiencia Provincial de Barcelona que preguntaba sobre la interpretación de la Directiva 93/13/CEE sobre las cláusulas abusivas en los contratos celebrados con consumidores. El Tribunal de Justicia solo se pronunció sobre dos cuestiones: si el hecho de que se siga un procedimiento monitorio impide que el juez examine de oficio una cláusula abusiva y qué debe hacer un juez ante una cláusula abusiva anularla con todas las consecuencias o integrarla en los términos que señala la legislación española.

El supuesto que originó el litigio era un contrato de préstamo para la compra de un vehículo que había fijado unos intereses de demora del 29\% y ante el impago de las cuotas Banesto inició un juicio monitorio. El Juzgado de Instancia de Sabadell anuló de oficio la cláusula de intereses moratorios y fijó los intereses de demora en un 19\%. No obstante, en apelación la Audiencia Provincial recurrió al Tribunal de Justicia.

Por lo que se refiere a la primera cuestión, el Tribunal de Justicia comprobó que el sistema procesal español no permite al juez nacional que conoce de una demanda en un proceso monitorio examinar de oficio en ninguna fase del procedimiento el carácter abusivo de una cláusula contenida en un contrato celebrado entre un profesional y un consumidor cuando este último no haya formulado oposición ni siquiera puede pronunciarse sobre 
si tal cláusula resulta contraria a las normas nacionales de orden público (apartado 48). Por esa razón y a juicio del Tribunal de Justicia la regulación procesal española es contraria al Derecho de la Unión en la medida en que «un régimen procesal de este tipo, que no permite que el juez que conoce de una demanda en un proceso monitorio, aun cuando ya disponga de todos los elementos de hecho y de Derecho necesarios al efecto, examine de oficio - in limine litis ni en ninguna fase del procedimiento- el carácter abusivo de las cláusulas contenidas en un contrato celebrado entre un profesional y un consumidor, cuando este último no haya formulado oposición, puede menoscabar la efectividad de la protección que pretende garantizar la Directiva 93/13» (apartado 53).

Del mismo modo y por lo que se refiere al alcance de la facultad del juez ante una cláusula abusiva, el Tribunal de Justicia determinar que no puede consistir, como exige la legislación española, en integrar dicho contrato modificando el contenido de la cláusula abusiva. En efecto, a juicio del Tribunal de Justicia: «incumbe a los tribunales nacionales que examinan el carácter abusivo de las cláusulas contractuales deducir todas las consecuencias que, según el Derecho nacional, se derivan de ello, a fin de evitar que las mencionadas cláusulas vinculen al consumidor» (apartado 63). Esto quiere decir que «el contrato en cuestión debe subsistir, en principio, sin otra modificación que la resultante de la supresión de las cláusulas abusivas, en la medida en que, en virtud de las normas del Derecho interno, tal persistencia del contrato sea jurídicamente posible» (apartado 65). En consecuencia y ante una cláusula abusiva, solo cabe suprimirla pero no cabe, como había hecho el Juez de instancia, reducir los intereses de demora del 29 al $19 \%$.

El Tribunal de Justicia responde en cuatro resoluciones a sendas cuestiones españolas contencioso-administrativas: tres proceden de la Sala de lo Contencioso-administrativo del Tribunal Supremo, por lo general con motivo del control de legalidad de Reales Decretos de transposición de Directivas europeas, y una de un Juzgado de lo Contencioso-administrativo de Valladolid que aplica la jurisprudencia comunitaria a la percepción de complementos retributivos de personal docente interino.

La sentencia Ascafor (C-484/10) se refiere a la libre circulación de productos de hormigón. En este caso la Instrucción de hormigón estructural, aprobada por el Real Decreto 1247/2008 e impugnada ante el Tribunal Supremo, restringía las posibilidades de importar acero para armaduras procedente de otros Estados miembros debido a las especificaciones exigidas.

El Tribunal de Justicia recuerda su jurisprudencia sobre la libre circulación de mercancías y señala: «en lo que respecta a los productos de construcción no cubiertos por especificaciones técnicas armonizadas o reconocidas a nivel de la Unión, los Estados miembros sólo pueden someter la comercialización en su territorio de dichos productos a disposiciones nacio- 
nales que respeten las obligaciones derivadas del Tratado, y en particular el principio de libre circulación de mercancías formulado en los artículos 34 TFUE y 36 TFUE» (apartado 50).

El Tribunal de Justicia constata que los requerimientos de certificación y de especificaciones técnicas son contrarios a la libre circulación porque disuaden a las empresas establecidas en España de importar acero para armaduras producido en otro Estado miembro y, sin embargo, no están justificadas por las razones de interés general enumeradas en el artículo 36 TFUE o por exigencias imperativas. En este caso, a juicio del Tribunal de Justicia, «el objetivo de protección de la salud y de la vida de las personas puede justificar tal obstáculo en la medida en que las exigencias impuestas no sobrepasen los requisitos mínimos establecidos para la utilización en España del acero para armar hormigón» lo que, ciertamente, no parecía ocurrir en este caso con la legislación española.

La sentencia Asociación Nacional de Expendedores de Tabaco y Timbre (ANETT) (C-456/10) también contesta una cuestión prejudicial sobre la libre circulación de mercancías, en particular, sobre la prohibición del Real Decreto 1/2007 de que los minoristas importen a España tabaco de otros Estados miembros.

En primer lugar y frente a la pretensión de la Comisión Europea y del Gobierno español de que la cuestión prejudicial se examinase a la luz del artículo 37 TFUE, sobre adaptación de los monopolios, y no respecto del artículo 34 TFUE, relativo a la libre circulación de mercancías, el Tribunal de Justicia consideró, como había explicado el Tribunal Supremo, que una normativa nacional que prohíbe a los minoristas de tabaco importar labores de tabaco debe examinarse en este caso de acuerdo con el artículo 34 TFUE.

En segundo lugar, el Tribunal de Justicia comprobó fácilmente que el Real Decreto español, que prohíbe a los minoristas de tabaco importar directamente dichos productos de otros Estados miembros y que los obliga a abastecerse a través de los mayoristas autorizados, constituye una medida de efecto equivalente a una restricción cuantitativa en el sentido del artículo 34 TFUE. Y, en fin, el Tribunal de Justicia consideró que la restricción a la libre circulación de mercancías no estaba justificada por razones fiscales, aduaneras y sanitarias, y que tampoco beneficiaba a los consumidores.

En su sentencia Génesis Seguros (C-190/10) el Tribunal Supremo preguntaba si en el caso del registro de marcas se pueden tener en cuenta, como ocurre en España, la hora y el minuto de presentación a los efectos de determinar la prioridad temporal de una marca nacional (ante la Oficina Española de Patentes y Marcas) respecto de una marca comunitaria (ante la Oficina de Armonización del Mercado Interior, OAMI).

Ahora bien, el Tribunal de Justicia explica: «el Reglamento n. ${ }^{\circ}$ 40/94 [sobre la marca comunitaria] debe interpretarse en el sentido de que no per- 
mite tomar en consideración - además del día - la hora y el minuto de presentación de la solicitud de marca comunitaria ante la OAMI, a fin de determinar la anterioridad de tal marca en relación con una marca nacional presentada el mismo día, aun cuando, en virtud de la normativa nacional [española] que regula el registro de esta última marca, la hora y el minuto de la presentación sean elementos pertinentes a este respecto».

El auto Lorenzo Martínez / Junta de Castilla y León (C-556/11) contesta un reenvío prejudicial planteado por el Juzgado de lo Contenciosoadministrativo n. ${ }^{\circ} 4$ de Valladolid respecto del derecho de los funcionarios interinos a la percepción de los denominados sexenios de formación de los profesores docentes no universitarios.

El Tribunal de Justicia responde la cuestión prejudicial reiterando los mismos argumentos de las sentencias de 13 de septiembre de 2007, Del Cerro Alonso (C-307/05), de 22 de diciembre de 2010, Gavieiro Gavieiro e Iglesias Torres (C-444/09 y C-456/09), auto de 18 de marzo de 2011, Montoya Medina (C-273/10), y de la sentencia de 8 de septiembre de 2011, Rosado Santana (C-177/10). Por lo que, del mismo modo que, en relación con los trienios del personal funcionario y del personal estatutario, a los sexenios del personal docente también se les aplica la Directiva 1999/70/CE del Consejo, de 28 de junio de 1999, sobre el trabajo de duración determinada.

Por tanto, no es conforme con el Derecho de la Unión la regulación estatal y autonómica que en España reserva, sin ninguna justificación por razones objetivas, el derecho a percibir el complemento retributivo por formación permanente únicamente a los profesores funcionarios de carrera, excluyendo a los profesores funcionarios interinos, cuando, en relación con la percepción de dicho complemento, ambas categorías de trabajadores se hallan en situaciones comparables.

\section{Los nuevos reenvíos prejudiciales españoles: los asuntos del «céntimo} sanitario» y sobre la protección de datos

De los nuevos reenvíos prejudiciales registrados en el Tribunal de Justicia en 2012 y procedentes de tribunales españoles es preciso destacar dos referidos a cuestiones de máxima actualidad y de gran trascendencia: los impuestos autonómicos sobre hidrocarburos, conocidos como «céntimo sanitario», y el derecho a la protección de los datos personales en Internet.

No obstante, siguen llegando reenvíos prejudiciales referidos a la igualdad de trabajadores masculinos y femeninos como consecuencia de un permiso de paternidad (asunto C-5/12, petición planteada por el Juzgado de lo Social de Lleida, Marc Betriu Montull / Instituto Nacional de la Seguridad 
Social) o respecto de la interpretación de la Directiva 1999/44/ sobre determinados aspectos de la venta y las garantías de los bienes de consumo en cuanto se refiere a defectos de escasa importancia (asunto C-32/12, petición del Juzgado de Primera Instancia de Badajoz, Soledad Duarte Hueros / Autociba S.A., Automóviles Citroen España S.A).

\subsection{LOS IMPUESTOS ESPECIALES SOBRE HIDROCARBUROS Y LAS COMUNIDADES AUTÓNOMAS («CÉNTIMO SANITARIO»): ASUNTO TRANSPORTES JORDI BESORA}

Mediante auto de 29 de noviembre de 2011 el Tribunal Superior de Justicia de Cataluña ha planteado al Tribunal de Justicia un reenvío prejudicial, asunto Transportes Jordi Besora, C-82/12, en el que suscita la duda de si el gravamen establecido por Cataluña en relación con el impuesto especial sobre ventas minoristas de hidrocarburos en los ejercicios 2005 a 2008 es conforme con el Derecho de la Unión Europea.

En el reenvío prejudicial se hace referencia a la Directiva 92/12/CE relativa al régimen general, tenencia, circulación y controles de los productos objeto de impuestos especiales, que ha sido sustituida por la Directiva 2008/118/CE relativa al régimen general de los impuestos especiales. Y subsidiariamente también se plantea si la regulación española es conforme con la legislación relativa al IVA.

La clave del reenvío prejudicial es la interpretación del artículo 3.2 de la Directiva 92/12 conforme al cual: «Los productos [hidrocarburos, alcohol y tabaco] podrán estar gravados por otros impuestos indirectos de finalidad específica, a condición de que tales impuestos respeten las normas impositivas aplicables en relación con los impuestos especiales o el IVA para la determinación de la base imponible, la liquidación, el devengo y el control del impuesto». Es importante señalar que la propia Comisión Europea había iniciado un procedimiento por incumplimiento contra España en este mismo ámbito y que llegó en 2008 a un dictamen motivado sin que la Comisión hubiese acudido luego al Tribunal de Justicia.

La interpretación que finalmente dé el Tribunal de Justicia tendrá una trascendencia presupuestaria extraordinaria dada la generalización en estos tiempos de crisis de este mecanismo de ingresos que se ha generalizado entre las Comunidades Autónomas españolas.

\subsection{LA PROFUNDIZACIÓN EN EL DERECHO A LA PROTECCIÓN DE LOS DATOS PERSONALES EN INTERNET: ASUNTO GOOGLE SPAIN}

El reenvío prejudicial formulado por la Audiencia Nacional al Tribunal de Justicia en el asunto Google Spain y Google / Agencia Española de Protección de Datos (C-131/12) también resulta muy relevante. 
El origen del litigio están en el ejercicio del «derecho al olvido» por parte de un ciudadano español, M.C., en relación con datos relativos a una subasta de inmuebles relacionada con un embargo derivado de deudas a la Seguridad Social, recogidos en La Vanguardia de Barcelona y que habían sido indexados por el buscador Google. El Sr. C. intentó que la empresa periodística y la empresa del buscador eliminasen sus datos pero en el caso de Google su reclamación fue derivada a la sede de la compañía en California. Finalmente, el Sr. C. denunció la situación a la Agencia Española de Protección de Datos que dictó la Resolución de 30 de julio de 2010 en la que se estimaba la reclamación contra Google Spain SL y contra Google Inc a las que instaba a que adoptasen las medidas necesarias para retirar los datos de su índice e impidiesen el acceso futuro; aunque, no obstante, inadmitió la reclamación formulada contra La Vanguardia Ediciones por considerar que había denegado de forma motivada la cancelación solicitada de los datos personales del afectado en la medida en que la publicación de los datos tenía justificación legal y su fin era dar la máxima publicidad a las subastas para conseguir la mayor concurrencia de licitadores. Google recurrió la resolución administrativa ante la Sala de lo Contencioso-administrativo de la Audiencia Nacional que el 27 de febrero de 2012 decidió plantear varias cuestiones al Tribunal de Justicia.

Las preguntas de la Audiencia Nacional se refieren a la aplicación territorial de la Directiva 95/46/CE sobre protección de datos personales y, consiguientemente de la normativa española de protección de datos, sobre la actividad de los buscadores como proveedores de contenidos, así como respecto del alcance del derecho de cancelación y de oposición en relación con el derecho al olvido. La sentencia resultante del Tribunal de Justicia podrá constituir, sin duda, un hito y una orientación precisamente en un momento en que se están debatiendo las propuestas legislativas presentadas en enero de 2012 por la comisaria Viviane Reding para modernizar las normas europeas sobre protección de datos.

15 de junio de 2012

\section{Relación de las sentencias comentadas}

1. TJ (Gran Sala), sentencia de 24 de enero de 2012, Maribel Domínguez (C-282/10) (derecho a las vacaciones y baja por enfermedad).

2. TJ (Sala 6. ${ }^{\mathrm{a}}$ ), auto de 9 de febrero de 2012, María Jesús Lorenzo Martínez / Junta de Castilla y León (C-556/11) (sexenios de profesores de enseñanza secundaria). 
3. TJ (Sala 1. ${ }^{\mathrm{a}}$ ), sentencia de 16 de febrero de 2012, Celaya Emparanza y Galdós Internacional, S.A. (C-488/10) (derecho exclusivo de utilización de un dibujo o modelo comunitario registrado).

4. TJ (Sala 3. ${ }^{\mathrm{a}}$ ), sentencia de 16 de febrero de 2012, Sabam / Netlog NV (C-360/10) (filtrado de datos personales en la plataforma de una red social).

5. TJ (Gran Sala), sentencia de 28 de febrero de 2012, Inter-Environnement Wallonie ASBL (Decreto valón) (C-41/11) (suspensión de los efectos de la anulación de un decreto valón en materia de aguas).

6. TJ (Sala 2. ${ }^{a}$ ), sentencia de 1 de marzo de 2012, Baris Akyüz (C-467/10) (denegación del permiso de conducir checo en Alemania).

7. TJ (Sala 5. ${ }^{\text {a }}$, sentencia de 1 de marzo de 2012, Ascafor (C-484/10) (libre circulación de productos de hormigón).

8. TJ (Sala 5. ${ }^{\text {) }}$, sentencia de 1 de marzo de 2012, Ángel Lorenzo González Alonso / Nationale Nederlanden Vida Cía. De Seguros y Reaseguros S.A.E (C-166/11) (venta de seguros fuera de un establecimiento comercial y protección del consumidor).

9. TJ (Sala 2. ${ }^{\text {a }}$, sentencia de 1 de marzo de 2012, Dermod Patrick O’Brien (C-393/10) (discriminación por la jubilación de los jueces a tiempo parcial).

10. TJ (Sala 3. ${ }^{\mathrm{a}}$ ), sentencia de 15 de marzo de 2012, Marco del Corso (C-135/10) (emisiones radiofónicas recibidas en la sala de espera de un dentista).

11. TJ (Sala 1. ${ }^{\text {a }}$, sentencia de 22 de marzo de 2012, Génesis Seguros Generales Sociedad Anónima de Seguros y Reaseguros (Génesis) (C-190/10) (cuestiones administrativas en el registro de marcas).

12. TJ (Gran Sala), sentencia de 22 de marzo de 2012, Inter-Environnement Wallonie ASBL (Orden bruselense) (C-567/10) (derogación de un plan de la ciudad de Bruselas y evaluación de impacto ambiental).

13. TJ (Sala 4. ${ }^{\mathrm{a}}$ ), sentencia de 29 de marzo de 2012, SAG ELV Slovensko (C-599/10) (contratación pública y petición de aclaración de ofertas).

14. TJ (Gran Sala) sentencia de 19 de abril de 2012, Bonnier Audio (C-461/10) (identificación de un usuario a Internet y protección de derechos de autor).

15. TJ (Sala 2. ${ }^{\mathrm{a}}$ ) sentencia de 19 de abril de 2012, Galina Meister (C-415/10) (discriminación por razón de sexo o de origen étnico en la selección de trabajadores).

16. TJ (Gran Sala), sentencia de 24 de abril de 2012, Servet Kamberaj (C-571/10) (discriminación de ciudadanos de terceros países residentes de larga duración en la Unión Europea). 
17. TJ (Sala $1 .^{\mathrm{a}}$ ) sentencia de 26 de abril de 2012, Nemzeti Fogyasztóvédelmi Hatóság (Oficina Nacional del Consumo) (C-472/10) (cláusulas abusivas de los contratos y acciones colectivas).

18. TJ (Sala 3. ${ }^{\text {a) }}$, sentencia de 26 de abril de 2012, DR, TV2 Danmark (C-510/10) (derechos de autor de grabaciones realizadas para televisión).

19. TJ (Sala 3.a), sentencia de 26 de abril de 2012, Asociación Nacional de Expendedores de Tabaco y Timbre (ANETT) (C-456/10) (libre circulación de mercancías y estancos).

20. TJ (Sala 2. ${ }^{\text {a) }}$, sentencia de 26 de abril de 2012, Wolfgang Hofmann (C-419/10) (permiso de conducir checo en Alemania).

21. TJ (Gran Sala), sentencia de 2 de mayo de 2012, SAS Institute Inc (C406/10) (protección de los derechos de autor de programas de ordenador).

22. TJ (Sala 3. ${ }^{\mathrm{a}}$ ), sentencia de 3 de mayo de 2012, España / Comisión (C-24/11 P) (anulación de correcciones financieras en ayudas al aceite de oliva).

23. TJ (Sala 2. ${ }^{\text {a }}$, sentencia de 10 de mayo de 2012, Duomo Gpa y otros (C-357/10 a C-359/10) (solvencia de licitadores y concesión de servicios tributarios municipales).

24. TJ (Sala 1. ${ }^{\mathrm{a}}$ ), sentencia de 14 de junio de 2012, Banesto (C-618/10) (cláusulas abusivas e intereses bancarios). 